\title{
Adsorption Behaviors of Cobalt on the Graphite and SiC Surface: A First-Principles Study
}

\author{
Wenyi Wang, ${ }^{1,2,3}$ Chuan Li, ${ }^{1,2,3}$ Jianzhu Cao, ${ }^{1,2,3}$ and Chao Fang ${ }^{1,2,3}$ \\ ${ }^{1}$ Institute of Nuclear and New Energy Technology, Tsinghua University, Beijing 100084, China \\ ${ }^{2}$ Collaborative Innovation Center of Advanced Nuclear Energy Technology, Beijing 100084, China \\ ${ }^{3}$ The Key Laboratory of Advanced Reactor Engineering and Safety, Ministry of Education, Beijing 100084, China
}

Correspondence should be addressed to Chao Fang; fangchao@tsinghua.edu.cn

Received 19 October 2016; Revised 13 February 2017; Accepted 12 March 2017; Published 20 March 2017

Academic Editor: Arkady Serikov

Copyright (C) 2017 Wenyi Wang et al. This is an open access article distributed under the Creative Commons Attribution License, which permits unrestricted use, distribution, and reproduction in any medium, provided the original work is properly cited.

\begin{abstract}
Graphite and silicon carbide $(\mathrm{SiC})$ are important materials of fuel elements in High Temperature Reactor-Pebble-bed Modules (HTR-PM) and it is essential to analyze the source term about the radioactive products adsorbed on graphite and $\mathrm{SiC}$ surface in HTR-PM. In this article, the adsorption behaviors of activation product Cobalt (Co) on graphite and SiC surface have been studied with the first-principle calculation, including the adsorption energy, charge density difference, density of states, and adsorption ratios. It shows that the adsorption behaviors of $\mathrm{Co}$ on graphite and $\mathrm{SiC}$ both belong to chemisorption, with an adsorption energy $2.971 \mathrm{eV}$ located at the Hollow site and $6.677 \mathrm{eV}$ located at the hcp-Hollow site, respectively. Combining the charge density difference and density of states, it indicates that the interaction of Co-SiC system is stronger than Co-graphite system. Furthermore, the variation of adsorption ratios of Co on different substrate is obtained by a model of grand canonical ensemble, and it is found that when the temperature is close to $650 \mathrm{~K}$ and $1700 \mathrm{~K}$ for graphite surface and $\mathrm{SiC}$ surface, respectively, the Co adatom on the substrate will desorb dramatically. These results show that $\mathrm{SiC}$ layer in fuel element could obstruct the diffusion of Co effectively in normal and accidental operation conditions, but the graphite may become a carrier of Co radioactivity nuclide in the primary circuit of HTR-PM.
\end{abstract}

\section{Introduction}

Cobalt-60 $\left({ }^{60} \mathrm{Co}\right)$ is a kind of long half-life $\gamma$-ray radionuclide and it could be generated through activation reaction of impurities $\left({ }^{59} \mathrm{Co}\right.$ and $\left.{ }^{60} \mathrm{Ni}\right)$ in the material of fuel elements and metal/nonmetal reactor internals of High Temperature Reactor-Pebble-bed Modules (HTR-PM). The behavior of ${ }^{60} \mathrm{Co}$ is important for the safety analysis and radiation protection design in HTR-PM and has attracted a lot of attentions. In the fuel element of HTR-PM, ${ }^{60} \mathrm{Co}$ in porous pyrolytic carbon (buffer layer) and dense inner pyrolytic carbon (IPyC) of TRISO fuel particle will diffuse and interact with silicon carbide ( $\mathrm{SiC}$ ) of TRISO fuel particle [1], which is considered to possibly influence the performance of SiC. In the primary circuit of HTR-PM, ${ }^{60} \mathrm{Co}$ on the surfaces of fuel elements, graphite reflectors, graphite reactor internals, and metal reactor internals will adsorb on the graphite dust, which is generated through abrasion or corrosion effect when the fuel elements flow in the primary circuit and is playing a significant role in contributing to the source term of HTR-PM [2]. It is reported that, in the end of lifetime of HTR-PM, the radioactivity of ${ }^{60} \mathrm{Co}$ in the primary circuit will be accumulated to $8.6 \times 10^{10} \mathrm{~Bq}$ [3], while the activity concentration of other radionuclides is at least two orders of magnitude lower than that of ${ }^{60} \mathrm{Co}$.

It is known that the study of interaction between $\mathrm{Co}$ and reactor material is essential but it is difficult to obtain the experimental result. Fortunately, the first-principle calculation, a powerful tool to study on an atomic scale, provides a way to research the above issue [4-7]. Based on the density functional theory (DFT) [8], the first-principle calculation is implemented in the Vienna Ab initio Simulation Package (VSAP) by the group of Kresse et al. [9-11], which could be used to study the atom-material interaction in microlevel [12-14]. A number of theoretical studies about the adsorption behaviors using first-principle calculations, especially 
for the graphite [15-19] and $\mathrm{SiC}$ nanotubes [20-27], have been published. Ancilotto and Toigo have performed firstprinciples total-energy calculations to study the adsorption of potassium on graphite [15]. Electronic structure calculations based on spin-polarized DFT with the generalized gradient approximation (GGA) and ultrasoft pseudopotentials are used to investigate the interaction between $\mathrm{H}$ atoms and a graphite surface [16]. The single Co atom adsorbed on some graphite materials also is discussed [28-31]. Wehling et al. have researched the orbitally controlled Kondo effect of Co adatoms on graphene [28]. Rudenko et al. have researched the adsorption of Co on graphene and analysis of the electron correlation effects from a quantum chemical perspective [29].

However, there is few works on the adsorption/desorption of $\mathrm{Co}$ on graphite/SiC and the mechanism of interaction is also not clear. In this work, the behavior of Co adsorbed on graphite and $\mathrm{SiC}$ surface with DFT will be studied, including the charge density difference (CDD) and the density of states (DOS). Furthermore, the mechanism of adsorption will also be discussed. At last, the variation of adsorption ratios of Co will be given by a model of grand canonical ensemble, which is significant for understanding the adsorption of Co on graphite and $\mathrm{SiC}$ macroscopically.

\section{Method of Calculation}

2.1. The Construction of Graphite and SiC Micromodel. The first-principle calculation is also known as ab initio calculation; it has been performed based on DFT as implemented in the VASP code and employed the projector augmented wave (PAW) pseudo-potential [32] and GGA-PBE exchangecorrelation functional [33] to describe the interaction of electron-ion. Since a large number of basis functions are usually required to describe the electronic wave functions appropriately, it is very demanding to use the first-principles pseudo-potential method to calculate the carbon properties [15]. To simplify the calculations, we choose a single, isolated graphite monolayer with $6 \times 6$ primitive cells (Figure 1(a)), by using a $K$-point mesh of $1 \times 1 \times 1$, as the substrate to calculate the adsorption energy. According to the anisotropic character of bonding in graphite, the coupling between adjacent graphite layers is much weaker than the in-plane coupling between carbon atoms $[14,34,35]$. This means that the electronic properties of a monolayer can usually provide a reasonable picture of the electronic properties of the infinite crystal [36]. As shown in Figure 1(b), graphite is here represented by graphene; there are three different adsorption sites on the graphene including Top (T), Bridge (B), and Hollow $(\mathrm{H})$. SiC used in TRISO fuel particle of fuel element in HTR-PM is $\beta$-SiC and the corresponding structure is diamond cubic crystal. Different from pure diamond, there are four silicon atoms surrounding carbon in each $\mathrm{SiC}$ crystal. Therefore, the interaction between adjacent atoms in a crystal is very strong and makes the structure of $\mathrm{SiC}$ especially stable, which is conducive to $\mathrm{SiC}$ to block the activation products diffuse from the fuel particle. The supersurface of $\mathrm{SiC}(001)$ lattice with $3 \times 3 \times 1$ primitive cells (Figure $2(\mathrm{a})$ ) and the $K$-point mesh as $2 \times 2 \times 1$ are employed to calculate the adsorption energy. The four kinds of adsorptive sites are including Top (T), Bridge (B), hcp-Hollow (hH), and fccHollow (fH) as shown in Figure 2(b).

2.2. Adsorption Energy. The adsorption energy of Co is defined as

$$
E_{\mathrm{ads}}=E_{\mathrm{Co}-\mathrm{sub}}-\left(E_{\mathrm{sub}}+E_{\mathrm{Co}}\right),
$$

where $E_{\mathrm{ads}}, E_{\mathrm{sub}}, E_{\mathrm{Co}}$, and $E_{\mathrm{Co} \text {-sub }}$ are the energies of the adsorption, substrate, a single Co atom, and the Co-substrate system, respectively. $E_{\text {sub }}, E_{\mathrm{Co}}$, and $E_{\mathrm{Co} \text {-sub }}$ could be obtained by the first-principle calculations directly, and then $E_{\mathrm{ads}}$ could be obtained from (1). There are two influencing factors that need to be considered which may affect the value of $E_{\text {ads }}$. One is the adsorptive sites and the other is the height between the adatom and substrate. The adsorption energies at different heights of different adsorptive sites are calculated firstly and after obtaining the most suitable position which located at the height corresponding to the largest adsorption energy, the total system will be relaxed to obtain the value of $E_{\mathrm{ads}}$ and obtain the optimum adsorptive site and height. It should be mentioned that the "-" sign of $E_{\mathrm{ads}}$ represents the direction of energy change, and the adsorption energy is only related to the absolute value of $E_{\text {ads }}$. Meanwhile, the CDD and the DOS could be obtained and are employed to analysis. The CDD is defined as the difference between the total charge density and the atomic charge densities [20]. It shows the visual image of electron transfer between nuclide and substrate directly. As suggested by Khantha et al. [37], the redistribution of the charge density indicates that the interaction potential between the adatom and substrate is composed of two distinct contributions: a screened Coulomb interaction resulting from the charge transfer between the adatom and substrate atoms and a van der Waals type interaction $[38,39]$. The equilibrium position of an adatom above graphite/ $\mathrm{SiC}$ is largely determined by the charge redistribution that takes place in this short-separation region. DOS shows the distributions of electrons in each energy level, which are helpful to understand the behaviors of atom absorbed/desorbed on material in microlevel. Fermi energy level $E_{F}$ could also be obtained in the abovementioned process. The relative position of the Fermi level in the DOS shows the occupation of the states and the features of bonding $[39,40]$. If atom is absorbed on the surface of substrate, the peaks of the state for both adatom and substrate compared to $E_{F}$ will be shifted. Supposing that the DOS is changed, the adsorption behavior between the adatom and substrate is chemisorption. Otherwise, it is physisorption. If the energy state of atom has a left shift, this means that the atom acquires electrons. Then its energy decreases, so that the peak width of state will become wider and more occupied. If the energy state shifted towards right, the atom will lose electrons.

What should be emphasized here is $E_{\mathrm{ads}}, \mathrm{CDD}, \mathrm{DOS}$, and $E_{F}$ are obtained in the condition of $0 \mathrm{~K}$ with DFT method, but the temperature condition of the fuel element and primary circuit of HTR-PM is below $1000 \mathrm{~K}$ under normal operation and below $1850 \mathrm{~K}$ under accident operation. It is necessary to point out that the energy of electron is not sensitive to the temperature at the range of $\mathrm{T} \sim 10^{3} \mathrm{~K}[41]$, so the results 


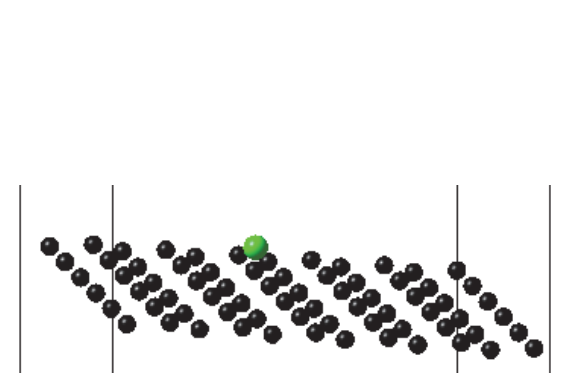

(a)

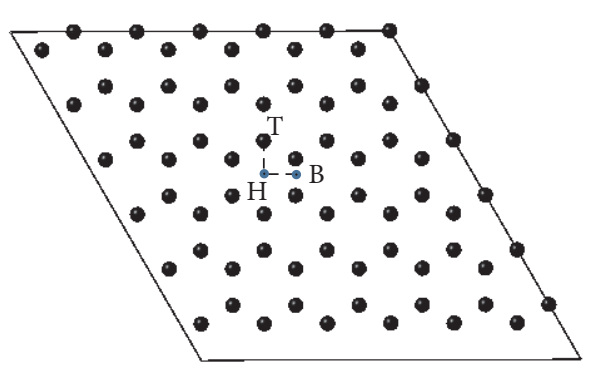

(b)

Figure 1: (a) The supersurface of Co on graphite. (b) The three adsorption sites on the graphite surface, where T, B, and $\mathrm{H}$ represent the Top, Bridge, and Hollow positions.

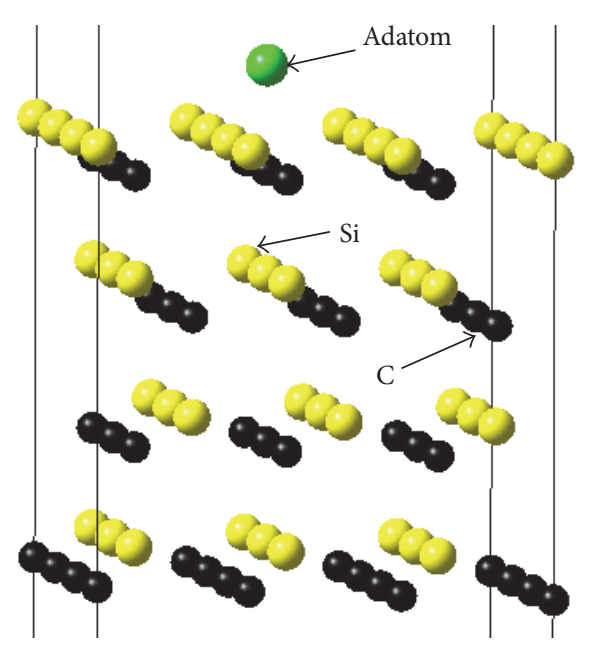

(a)

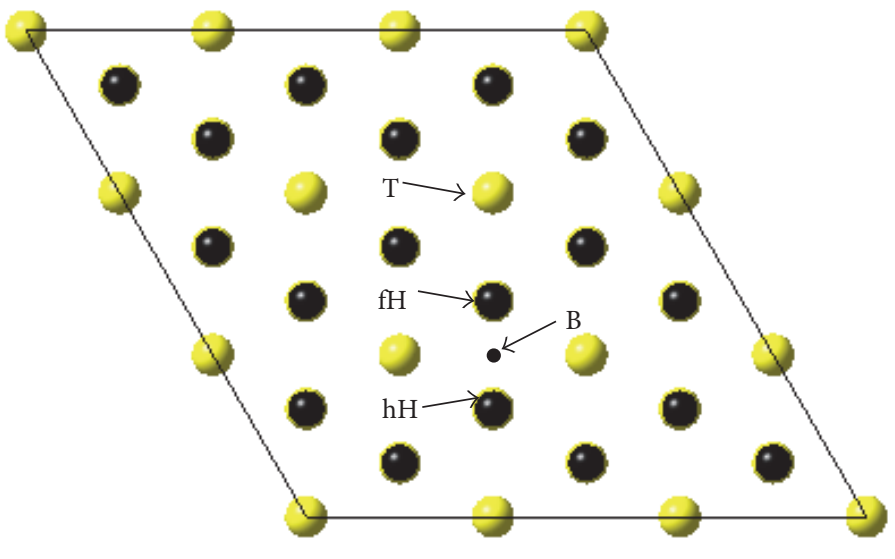

(b)

Figure 2: (a) The supersurface of Co on SiC. (b) The adsorption sites on the SiC surface, where T, B, and H represent the Top, Bridge, and Hollow positions.

obtained by DFT can be applied to study the interaction between Co and graphite and SiC of HTR-PM.

2.3. Adsorption Ratio. The adsorbed nuclide atoms on substrate and separate nuclide atoms will form a dynamic equilibrium system. According to literatures [14, 42], a model of grand canonical ensemble is considered to describe this system and calculate the balanced adsorption ratio of Co on the graphite and $\mathrm{SiC}$. In this model, the substrate materials adsorbing Co atoms are considered as an open system, and the separate Co atoms in circumstance (i.e., the coolant in the primary circuit of HTR-PM and the gas interlayer between $\mathrm{SiC}$ and IPyC of TRISO fuel particle) are treated as particle and gas source. On the theory of the statistic physics, the relation between the number of adsorbed Co atoms $\bar{N}$ and the total adsorptive centers $N_{0}$ is given by

$$
\bar{N}=\frac{N_{0}}{1+e^{\left(E_{\mathrm{ads}}-\mu\right) / K_{b} T}},
$$

where $\mu$ is the chemical potential of Co atom, $K_{b}$ is Boltzmann's constant, and $T$ is the temperature of the circumstance. So the adsorption ratio can be defined as

$$
\theta=\frac{\bar{N}}{N_{0}} .
$$

Because the concentration of radioactive products is very low [42], the ideal gas model can be employed to deal with nuclide gas source directly. If an equilibrium state is achieved in the reactor, the chemical potentials of nuclide atoms in the open system will be identical to those of gas source. Therefore, according to the ideal gas model, the chemical potential of Co can be written as follows:

$$
\mu=K_{b} T \ln \left[n\left(\frac{h^{2}}{2 \pi m K_{b} T}\right)^{3 / 2}\right],
$$

where $n$ is the density of the nuclide in the circumstance, $m$ is the atomic mass of Co, and $h$ is the Planck constant.

Combined with (2), (3), and (4), the adsorption ratio is accessible to obtain as

$$
\theta=\frac{1}{1+(1 / n)\left(2 \pi m K_{b} T / h^{2}\right)^{3 / 2} e^{E_{\mathrm{ads}} / K_{b} T}}
$$

It is seen from (5) that only three parameters of $n, T$, and $E_{\text {ads }}$ are undetermined. The numerical value of $n$ and $T$ 


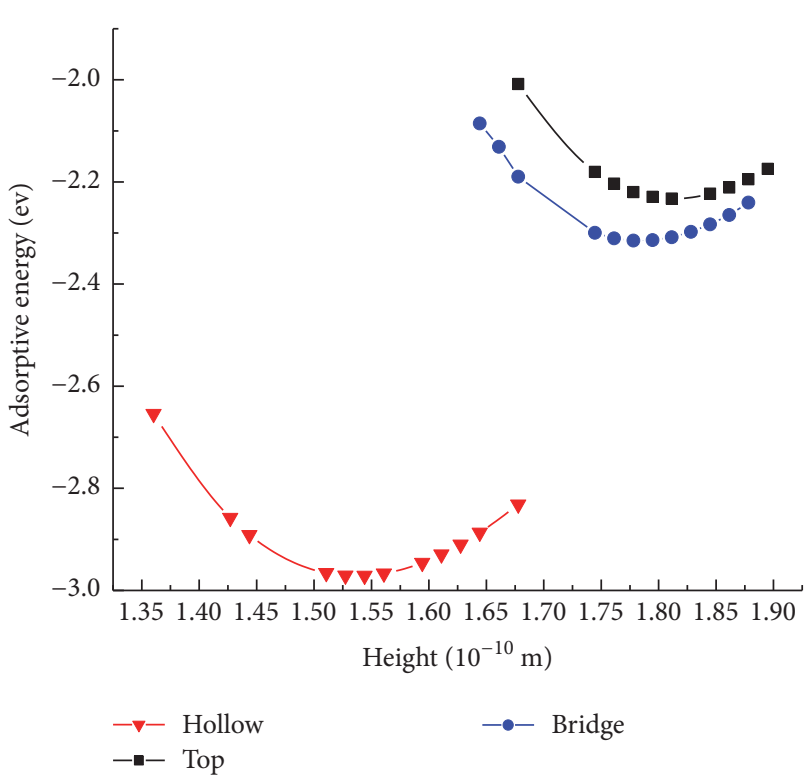

(a)

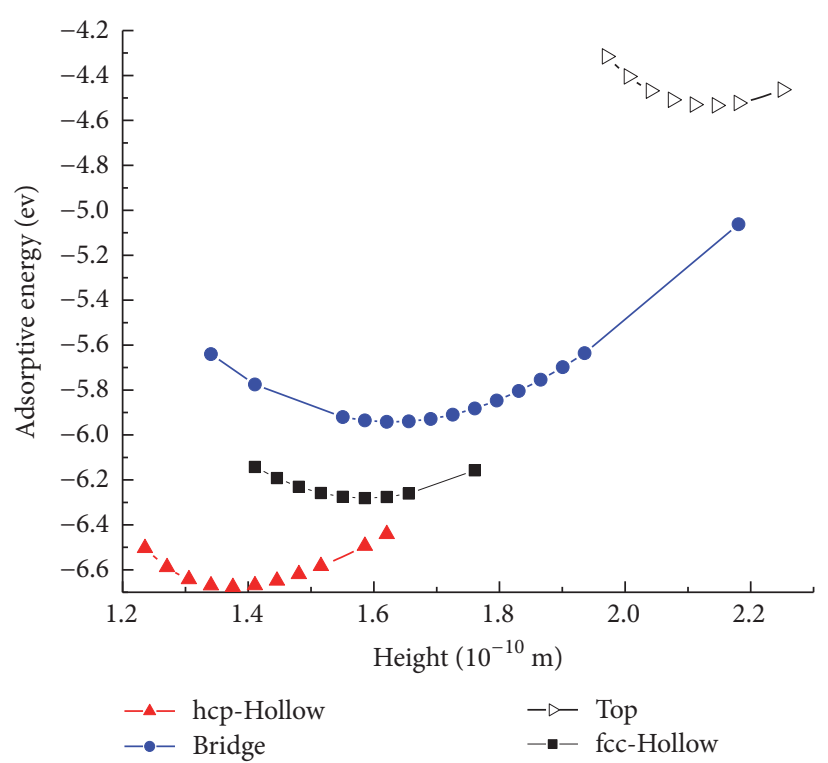

(b)

FIGURE 3: The variations of adsorption energies at different sites with different height. (a) The Co adatom at graphite. (b) The Co adatom at $\mathrm{SiC}$.

of circumvents in HTR-PM can be determined by either calculation or experimental measurement and $E_{\text {ads }}$ can be obtained from (1).

\section{Results and Discussion}

3.1. Adsorption Energy. In order to obtain the most stable adsorptive site, the adsorption energy with different adsorptive sites has been calculated. For each adsorptive site, the adsorption energy depends on the height between the adatom and substrate and there exists a maximum value which could be considered as the exact adsorption energy of corresponding site. Figure 3 shows the variations of adsorption energies of $\mathrm{Co}$ on graphite and $\mathrm{SiC}$ at different sites with different height.

The adsorption energies of $\mathrm{Co}$ on graphite and $\mathrm{SiC}$ at different adsorptive sites are listed in Tables 1 and 2. To different substrate, the adsorption energies and the most stable adsorptive sites of $\mathrm{Co}$ are different. It shows that the most stable sites for Co-graphite and $\mathrm{Co}-\mathrm{SiC}$ system are Hollow and hcp-Hollow with the adsorption energies $2.971 \mathrm{eV}$ and $6.677 \mathrm{eV}$, respectively. These results indicate that the interaction of $\mathrm{Co}-\mathrm{SiC}$ system is stronger than $\mathrm{Co}$-graphite system.

3.2. Analysis of Electronic Structure. To identify the adsorption behavior of the Co adatom on different substrates, the electronic structure of the two systems should be analyzed. Figure 4 shows the optimized stable configurations and their corresponding CDD for the Co-graphite system and Co$\mathrm{SiC}$ system. The blue areas represent the decreases of charge density and the yellow areas represent the increase of charge density. Even though there is no obvious electron transfer
TABLE 1: The adsorption energies at different adsorptive sites for the Co adatom on graphite.

\begin{tabular}{lcc}
\hline Adsorption sites & $E_{\text {ads }}(\mathrm{eV})$ & $d_{\mathrm{Co}-\mathrm{SiC}}(\AA)$ \\
\hline Bridge (B) & -2.315 & 1.778 \\
Top (T) & -2.233 & 1.811 \\
Hollow & $-\mathbf{2 . 9 7 1}$ & 1.544 \\
\hline
\end{tabular}

TABLE 2: The adsorption energies at different adsorptive sites for the Co adatom on $\mathrm{SiC}$.

\begin{tabular}{lcc}
\hline Adsorption sites & $E_{\text {ads }}(\mathrm{eV})$ & $d_{\mathrm{Co}-\mathrm{SiC}}(\AA)$ \\
\hline Bridge (B) & -5.942 & 1.621 \\
Top (T) & -4.533 & 2.146 \\
fcc-Hollow & -6.281 & 1.586 \\
hcp-Hollow & $-\mathbf{6 . 6 7 7}$ & 1.376 \\
\hline
\end{tabular}

between Co and graphite in Figure 4(a), the adsorption behavior of the Co-graphite system could be considered as chemisorption due to its high adsorption energy in Table 1, while in Figure 4(b) there are a lot of yellow areas that appeared between $\mathrm{Co}$ and $\mathrm{SiC}$ surface, which suggests that a strong chemical bond is formed in this area. This result indicates that the Co has a stronger interaction with $\mathrm{SiC}$ than graphite. The result also coincides with the abovementioned adsorption energies of these two systems.

In order to understand the deep features of interactions between the adatom and substrates, the DOS for single $\mathrm{Co}$ atom, the substrate of graphite and $\mathrm{SiC}$, and the Cosubstrate system are calculated. It should be mentioned that the $K$-point meshes to integrate the Brillouin zone have been changed to $2 \times 2 \times 2$ and $4 \times 4 \times 1$ for the DOS calculation 


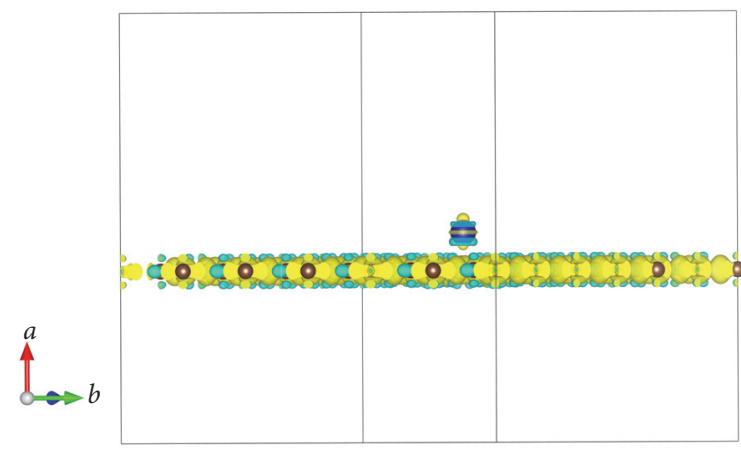

(a)

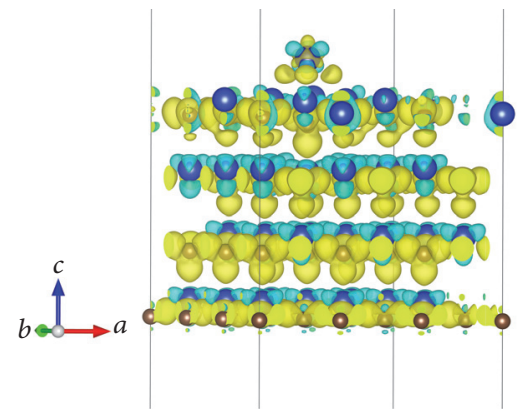

(b)

Figure 4: The CDD of Co at different substrate. (a) The Co adatom at graphite. (b) The Co adatom at SiC.

of Co-graphite system and Co-SiC system, respectively. The results of DOS are shown in Figure 5. When the Co adsorbed on graphite and $\mathrm{SiC}$ surface, the positions of Co adatom are different. In the following discussion, different systems and different positions corresponding to the adsorption state are used in the calculation of isolated Co atom for Co-graphite and $\mathrm{Co}-\mathrm{SiC}$, respectively, which makes the DOS of Co have some differences in these two systems.

Figures 5(a) and 5(b) give a comparison of DOS for the Co-graphite system, the isolated graphite substrate, the isolated Co atom, and the adsorbed Co adatom. For isolated Co atom, the peaks of the $d$-state nearly locate at $E_{F}$ and that of $s$-state locate at the right of $E_{F}$. When the Co atom is adsorbed on the graphite surface, the peaks of the $d$-state have a left shift towards a lower energy than $E_{F}$ and have evident broadenings owing to adsorption interaction. It is also found that the peak of the $s$-state shifts slightly right and the peak intensity becomes too weak to be identified when the Co atom is adsorbed on the graphite surface. Combining the above results and total DOS of graphite, it is feasible to know that the interaction between Co and graphite is very strong and there are electrons transferring on the interactive interface, which is the direct evidence of chemisorption. The situation of the Co-SiC system, as shown in Figures 5(c) and 5(d), is a little different. When the $\mathrm{Co}$ atom is adsorbed on the $\mathrm{SiC}$ surface, the peak intensities of the $s$-state become too weak to recognize and there are only shifts and broadenings of the peaks of $d$-state. Remarkably, although most of the peaks of $d$ state move to the left, there is still right shift, which indicates that $\mathrm{Co}$ adatom not only gains partial electrons from $\mathrm{SiC}$ but also loses electrons to $\mathrm{SiC}$. These results could be explained from the view of electrochemistry that the electronegativity of Co is close to Si and there exists resonance between the $d$ state of $\mathrm{Co}$ adatom and $\mathrm{SiC}$ surface when the partial electron transferring turns out. That is to say, due to the orbital hybridization, the adsorptive behavior between $\mathrm{Co}$ and $\mathrm{SiC}$ is also chemisorption. Based on the electronic analysis, it presented that the interaction between $\mathrm{Co}$ and $\mathrm{SiC}$ is stronger than that between Co and graphite significantly.

3.3. Adsorption Ratio. The adsorption ratio, as shown in (4), is determined by three parameters $n, T$, and $E_{\text {ads }}$. Among them, $E_{\text {ads }}$ can be obtained from (1) by the first-principle calculation, the temperature $T$ is variational, and the density of Co $(n)$ in circumstance could be obtained by experiment or theoretical calculations. Considering that the adsorption conditions of ${ }^{60} \mathrm{Co}$ on graphite and $\mathrm{SiC}$ surface are different in HTR-PM, the density of ${ }^{60} \mathrm{Co}$ used for calculation is not the same. Based on the experimental data, the density of ${ }^{60} \mathrm{Co}$ in the primary circuit could be set to be $2.4 \times$ $10^{10} \mathrm{~m}^{-3}$ [3]. The density of ${ }^{60} \mathrm{Co}$ on the surface of the SiC was calculated according to the content of ${ }^{59} \mathrm{Co}$ impurity in the graphite matrix, and the calculated result was $6.33 \times$ $10^{14} \mathrm{~m}^{-3}$. In order to investigate the trend of adsorption ratio varying with temperature $T$, the adsorption ratios of Co adatom on graphite and $\mathrm{SiC}$ surface calculated by (4) are shown in Figure 6. It can be easily noted that the adsorption ratio of the Co decreases exponentially with the increase of temperature.

According to Figure 6, there exists an inflexion point while the adsorption ratio varies with the temperature. When the temperature is close to $650 \mathrm{~K}$ and $1700 \mathrm{~K}$ for graphite surface and $\mathrm{SiC}$ surface, respectively, the adsorption ratio of Co decreases dramatically. The temperature at the inflexion point is suggested to be defined as the critical temperature, $T_{c}$. If the temperature is lower than $T_{c}$, most or even all of Co will be absorbed on the substrate surface. If the temperature is higher than $T_{c}$, it stands for the idea that the Co on the substrate will have a lot of transitions from absorption to desorption.

In HTR-PM, the average temperature of the fuel element and the primary circuit under normal operation is usually less than $1000 \mathrm{~K}$, and the maximum design value of temperature under accident conditions is about $1850 \mathrm{~K}$. From these data, it shows that Co could be adsorbed well on the surface of $\mathrm{SiC}$ in the normal operating temperature range and only at the highest temperature in supposed accidents, Co will be desorbed and diffused from the $\mathrm{SiC}$ surface. For graphite, Co is susceptible to a large amount of desorption and diffusion from the graphite surface whether in normal operation or accident conditions. As a result, the Co absorbed on $\mathrm{SiC}$ surface performed a better adsorption behavior than on graphite surface. Therefore, $\mathrm{SiC}$ could block the diffusion of Co, while graphite could not if the temperature is higher than $650 \mathrm{~K}$. 


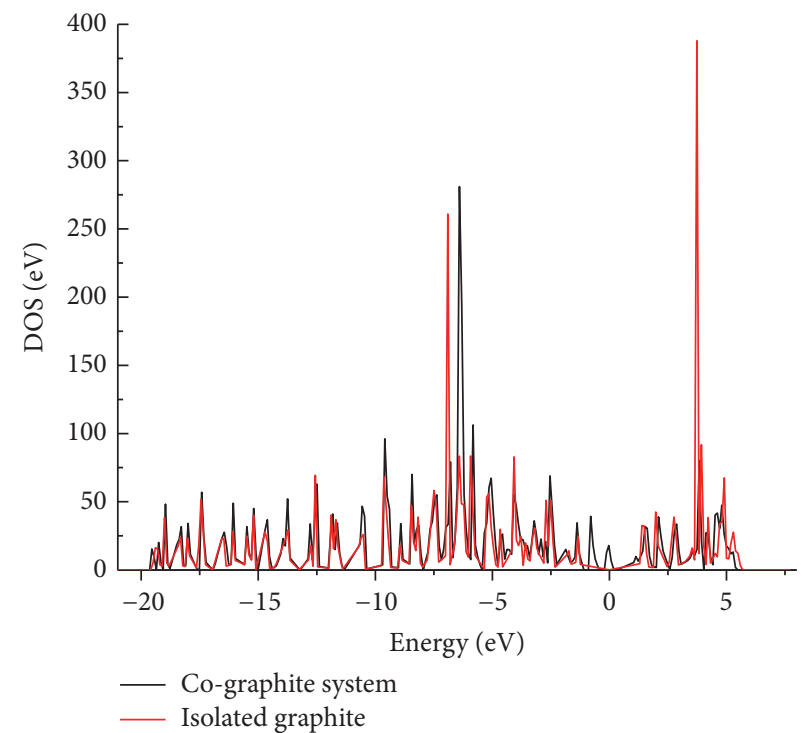

(a)

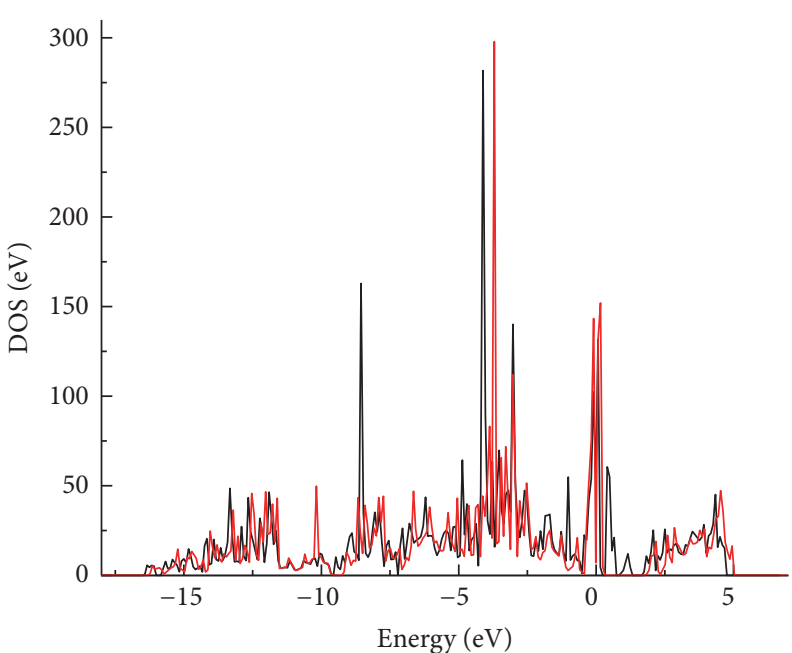

- Co-SiC system — Isolated $\mathrm{SiC}$

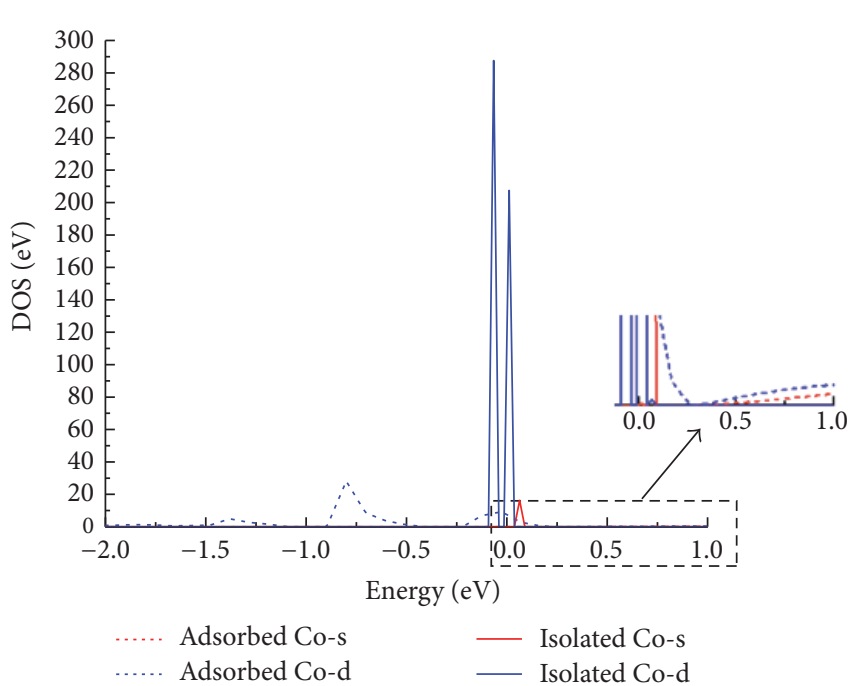

(b)

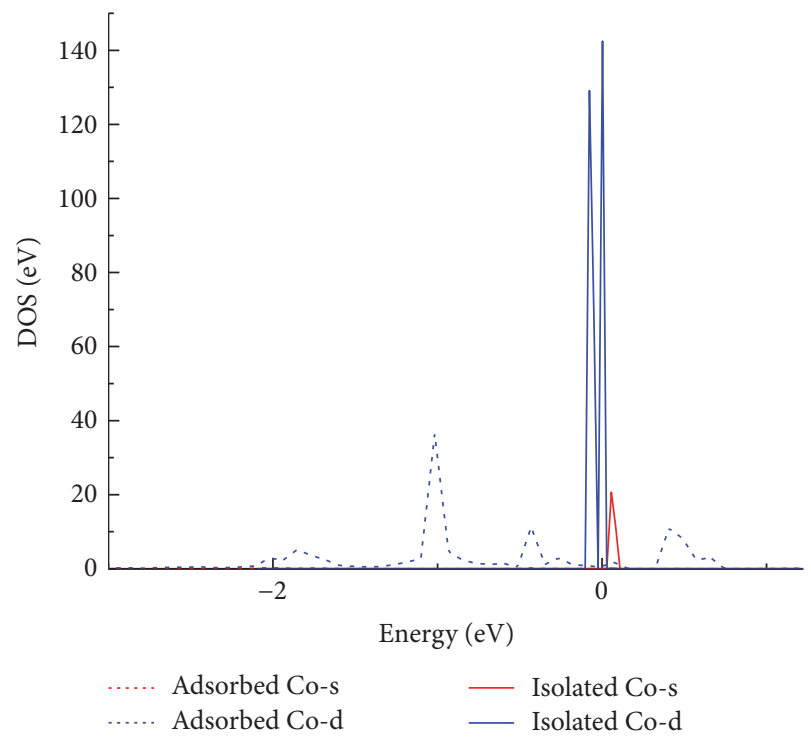

(d)

FIgURE 5: A comparison of DOS with Co: (a) the Co-graphite system and the isolated graphite substrate, (b) the isolated Co atom and the adsorbed $\mathrm{Co}$ adatom on the graphite, (c) the $\mathrm{Co}-\mathrm{SiC}$ system and the isolated $\mathrm{SiC}$ substrate, and (d) the isolated Co atom and the adsorbed $\mathrm{Co}$ adatom on the $\mathrm{SiC}$. (The number on the abscissa axes indicates the energy difference between the electronic state and the Fermi energy.)

\section{Conclusion}

In this paper, the first-principles DFT theory was used to study the adsorption behavior of Co on graphite and $\mathrm{SiC}$ surface in HTR-PM. The adsorption energies corresponding to different adsorptive site and height were calculated, and the adsorption energy for Co on graphite and $\mathrm{SiC}$ surface is $2.971 \mathrm{eV}$ and $6.677 \mathrm{eV}$, respectively. In order to know the underlying physical nature of the adsorption behavior of $\mathrm{Co}$, we have analyzed the electronic structure of the adsorption system by CDD and DOS. The results suggested that the adsorptive behavior of the two systems belongs to chemisorption. All of these results could be used to explain the result of adsorption energy from different aspects; the source term from the microscopic mechanism has been analyzed; it helps us to understand the adsorption behavior of the Co more clearly. In addition, combining the adsorption energy obtained by the first-principle calculation, the adsorption ratio was derived with different temperature and the density of Co in HTR-PM. The critical temperature for graphite surface is $650 \mathrm{~K}$, and for $\mathrm{SiC}$ surface it is $1700 \mathrm{~K}$.

In a conclusion, the adsorption performance of $\mathrm{Co}$ on the $\mathrm{SiC}$ surface was better than that on the graphite surface. In the primary circuit of HTR-PM, the Co adatom seems to be easily desorbed from the graphite surface. Compared with graphite, SiC could suppress the diffusion of Co effectively for both the normal operation and the accident conditions in the fuel elements. The results of adsorption ratios can also be 


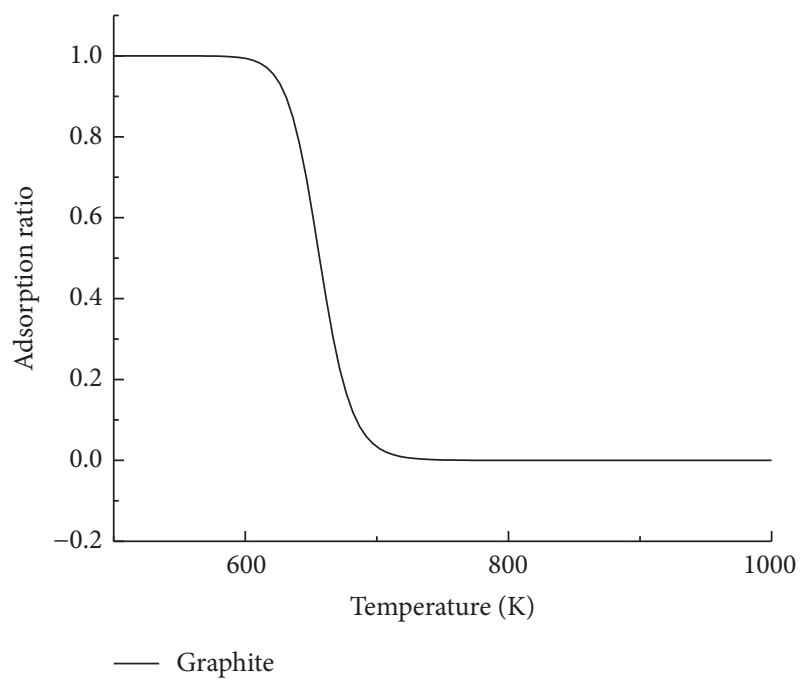

(a)

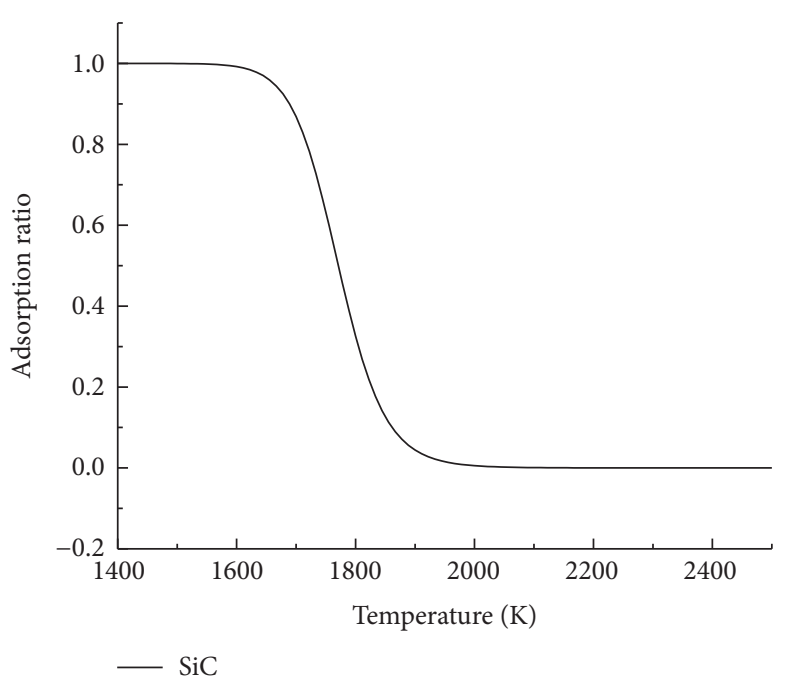

(b)

Figure 6: Variation of adsorption ratio with temperature for Co atom on (a) graphite and (b) SiC.

used for nuclear safety evaluation of Co release in the normal operation and the supposed accident of the HTR-PM.

\section{Conflicts of Interest}

The authors declare that there are no conflicts of interest regarding the publication of this paper.

\section{Acknowledgments}

This work was supported by the National Science and Technology Major Project of the Ministry of Science and Technology of China (Grant no. ZX06901).

\section{References}

[1] International Atomic Energy Agency, "Fuel performance and fission product behaviour in gas cooled reactors," Tech. Rep. IAEA-TECDOC-978, International Atomic Energy Agency, Vienna, Austria, 1997.

[2] C. Yang, C. Fang, and J. Cao, "The design and thermohydraulics study of the HTR-10 high temperature helium experimental loop," Progress in Nuclear Energy, vol. 77, pp. 329-335, 2014.

[3] L. Yuanzhong and C. Jianzhu, "Fission product release and its environment impact for normal reactor operations and for relevant accidents," Nuclear Engineering and Design, vol. 218, no. $1-3$, pp. 81-90, 2002.

[4] D. M. Riffe, G. K. Wertheim, and P. H. Citrin, "Alkali metal adsorbates on W(110): ionic, covalent, or metallic?" Physical Review Letters, vol. 64, no. 5, pp. 571-574, 1990.

[5] B. N. J. Persson and H. Ishida, "Depolarization and metallization in alkali-metal overlayers," Physical Review B, vol. 42, no. 5, pp. 3171-3174, 1990.

[6] K. Horn, A. Hohlfeld, J. Somers, T. Lindner, P. Hollins, and A. M. Bradshaw, "Identification of the s-derived valence-electron level in photoemission from alkali-metal adlayers on aluminum," Physical Review Letters, vol. 61, no. 21, pp. 2488-2491, 1988.
[7] G. M. Lamble, R. S. Brooks, D. A. King, and D. Norman, "Determination of an adlayer bonding transition by surface extended X-ray-absorption fine-structure spectroscopy: Cesium adsorbed on Ag 111," Physical Review Letters, vol. 61, no. 9, pp. 1112-1115, 1988.

[8] W. Kohn and L. J. Sham, "Self-consistent equations including exchange and correlation effects," Physical Review, vol. 140, no. 4, pp. A1133-A1138, 1965.

[9] G. Kresse and J. Furthmüller, "Efficient iterative schemes for ab initio total-energy calculations using a plane-wave basis set," Physical Review B, vol. 54, no. 16, pp. 11169-11186, 1996.

[10] G. Kresse and J. Furthmüller, "Efficiency of ab-initio total energy calculations for metals and semiconductors using a plane-wave basis set," Computational Materials Science, vol. 6, no. 1, pp. 15-50, 1996.

[11] G. Kresse and J. Hafner, "Ab initio molecular dynamics for liquid metals," Physical Review B, vol. 47, no. 1, pp. 558-561, 1993.

[12] X. Luo, C. Fang, X. Li, W. Lai, and T. Liang, "Adsorption behaviors of Cs and I atoms on the graphite surface by the firstprinciples," Journal of Nuclear Materials, vol. 441, no. 1, pp. 113118, 2013.

[13] X.-F. Luo, C. Fang, X. Li, W.-S. Lai, L.-F. Sun, and T.-X. Liang, "Adsorption and electronic structure of Sr and Ag atoms on graphite surfaces: a first-principles study," Chinese Physics Letters, vol. 30, no. 6, Article ID 066801, 2013.

[14] X. Luo, C. Fang, X. Li, W. Lai, L. Sun, and T. Liang, "Study of interaction between radioactive nuclides and graphite surface by the first-principles and statistic physics," Applied Surface Science, vol. 285, pp. 278-286, 2013.

[15] F. Ancilotto and F. Toigo, "First-principles study of potassium adsorption on graphite," Physical Review B, vol. 47, no. 20, pp. 13713-13721, 1993.

[16] X. Sha and B. Jackson, "First-principles study of the structural and energetic properties of $\mathrm{H}$ atoms on a graphite (0001) surface," Surface Science, vol. 496, no. 3, pp. 318-330, 2002.

[17] Y. Ferro, F. Marinelli, and A. Allouche, "Density functional theory investigation of the diffusion and recombination of $\mathrm{H}$ 
on a graphite surface," Chemical Physics Letters, vol. 368, no. 56, pp. 609-615, 2003.

[18] S. D. Chakarova-Käck, E. Schröder, B. I. Lundqvist, and D. C. Langreth, "Application of van der Waals density functional to an extended system: adsorption of benzene and naphthalene on graphite," Physical Review Letters, vol. 96, no. 14, Article ID 146107, 2006.

[19] S. D. Chakarova-Käck, Ø. Borck, E. Schröder, and B. I. Lundqvist, "Adsorption of phenol on graphite(0001) and $\alpha$ $\mathrm{Al}_{2} \mathrm{O}_{3}$ (0001): nature of van der Waals bonds from firstprinciples calculations," Physical Review B, vol. 74, no. 15, Article ID 155402, 2006.

[20] T. Meng, C.-Y. Wang, and S.-Y. Wang, "First-principles study of a single $\mathrm{Ti}$ atom adsorbed on silicon carbide nanotubes and the corresponding adsorption of hydrogen molecules to the $\mathrm{Ti}$ atom," Chemical Physics Letters, vol. 437, no. 4-6, pp. 224-228, 2007.

[21] G. Gao, S. H. Park, and H. S. Kang, "A first principles study of $\mathrm{NO}_{2}$ chemisorption on silicon carbide nanotubes," Chemical Physics, vol. 355, no. 1, pp. 50-54, 2009.

[22] E. Bekaroglu, M. Topsakal, S. Cahangirov, and S. Ciraci, "Firstprinciples study of defects and adatoms in silicon carbide honeycomb structures," Physical Review B, vol. 81, no. 7, Article ID 075433, 2010.

[23] E. F. Rosso and R. J. Baierle, "First principles study about Fe adsorption on planar SiC nanostructures: monolayer and nanoribbon," Microelectronic Engineering, vol. 126, pp. 37-41, 2014.

[24] Y.-L. Shi, J.-M. Zhang, and K.-W. Xu, "Structural and electronic properties of $\mathrm{SiC}$ nanotubes filled with $\mathrm{Cu}$ nanowires: a firstprinciples study," Physica E: Low-Dimensional Systems and Nanostructures, vol. 54, pp. 319-325, 2013.

[25] L. Wang, "Adsorption of formaldehyde $(\mathrm{HCOH})$ molecule on the SiC sheet: a first-principles study," Applied Surface Science, vol. 258, no. 17, pp. 6688-6691, 2012.

[26] P. V. Bui, K. Inagaki, Y. Sano, K. Yamauchi, and Y. Morikawa, "Adsorption of hydrogen fluoride on SiC surfaces: a density functional theory study," Current Applied Physics, vol. 12, no. 3, pp. S42-S46, 2012.

[27] M. Şimşek and S. Aydn, "First-principles study of thiophene on $\beta$-SiC ( $\left.\begin{array}{lll}0 & 0 & 1\end{array}\right)-(2 \times 1)$ surface," Physica B: Condensed Matter, vol. 406, no. 14, pp. 2880-2884, 2011.

[28] T. O. Wehling, A. V. Balatsky, M. I. Katsnelson, A. I. Lichtenstein, and A. Rosch, "Orbitally controlled Kondo effect of Co adatoms on graphene," Physical Review B, vol. 81, no. 11, Article ID 115427, 2010.

[29] A. N. Rudenko, F. J. Keil, M. I. Katsnelson, and A. I. Lichtenstein, "Adsorption of cobalt on graphene: electron correlation effects from a quantum chemical perspective," Physical Review B, vol. 86, no. 7, pp. 3305-3307, 2012.

[30] D. Krychowski, J. Kaczkowski, and S. Lipinski, "Kondo effect of a cobalt adatom on a zigzag graphene nanoribbon," Physical Review B, vol. 89, no. 3, Article ID 035424, 2014.

[31] H. Johll, H. C. Kang, and E. S. Tok, "Density functional theory study of $\mathrm{Fe}, \mathrm{Co}$, and $\mathrm{Ni}$ adatoms and dimers adsorbed on graphene," Physical Review B, vol. 79, no. 24, Article ID 245416, 2009.

[32] G. Kresse and D. Joubert, "From ultrasoft pseudopotentials to the projector augmented-wave method," Physical Review B, vol. 59, no. 3, pp. 1758-1775, 1999.
[33] J. P. Perdew, K. Burke, and M. Ernzerhof, "Generalized gradient approximation made simple," Physical Review Letters, vol. 77, no. 18, pp. 3865-3868, 1996.

[34] H. Ishida and R. E. Palmer, "Electronic structure and phase transitions of submonolayer potassium on graphite," Physical Review B, vol. 46, no. 23, pp. 15484-15489, 1992.

[35] S. Srivastava and J. Almlöf, "Chemisorption of aluminum atoms on a graphite surface: cluster convergence and effects of surface reconstruction," Surface Science, vol. 274, no. 1, pp. 113-119, 1992.

[36] S. B. Trickey, F. Müller-Plathe, G. H. F. Diercksen, and J. C. Boettger, "Interplanar binding and lattice relaxation in a graphite dilayer," Physical Review B, vol. 45, no. 8, pp. 44604468, 1992.

[37] M. Khantha, N. A. Cordero, L. M. Molina, J. A. Alonso, and L. A. Girifalco, "Interaction of lithium with graphene: an ab initio study," Physical Review B, vol. 70, no. 12, Article ID 125422, 2004.

[38] Y. Andersson, E. Hult, H. Rydberg, P. Apell, B. I. Lundqvist, and D. C. Langreth, "Van der Waals interactions in density functional theory," in Electronic Density Functional Theory, pp. 243-260, Springer, Berlin, Germany, 1998.

[39] Y. Mao, J. Yuan, and J. Zhong, "Density functional calculation of transition metal adatom adsorption on graphene," Journal of Physics Condensed Matter, vol. 20, no. 11, Article ID 115209, 2008.

[40] R. Pentcheva and M. Scheffler, "Initial adsorption of Co on $\mathrm{Cu}$ (001): a first-principles investigation," Physical Review B, vol. 65, no. 15, Article ID 155418, 2002.

[41] J. X. Fang and D. Lu, Solid Physics, Shanghai Scientific and Technical Publishers, Shanghai, China, 1980.

[42] C. Fang, J.-Z. Cao, and Y.-J. Dong, "Study of adsorption on graphite and its mechanism of radionuclides in primary circuit of HTR-10 with Ab initio theory," Atomic Energy Science and Technology, vol. 46, no. 1, pp. 391-396, 2012. 

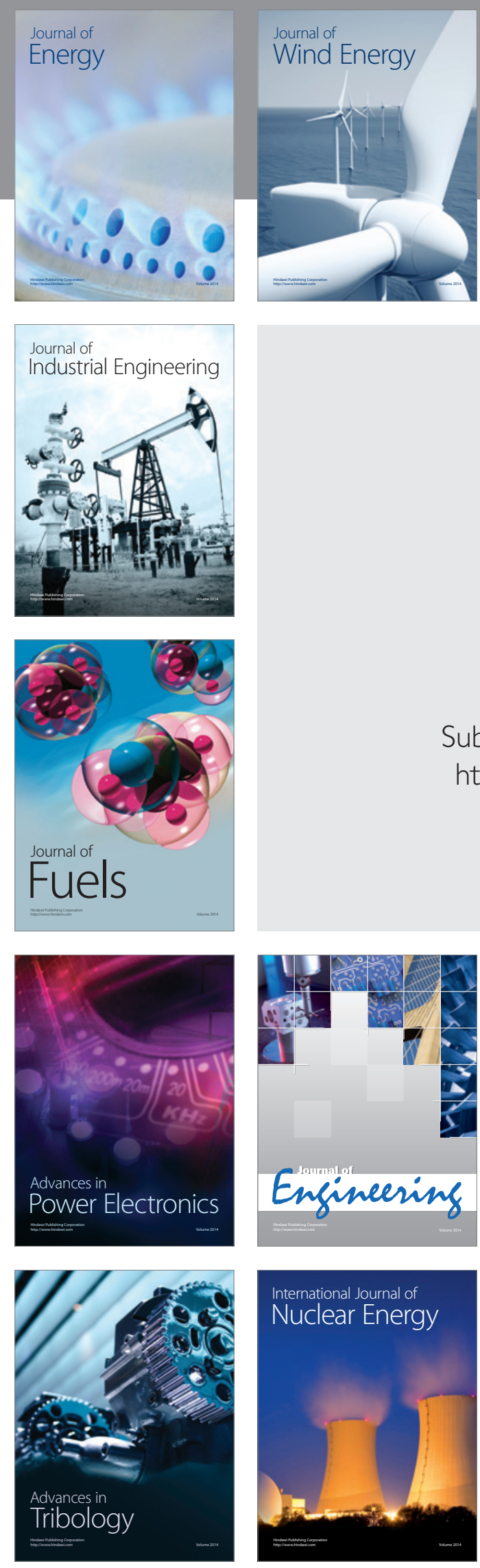

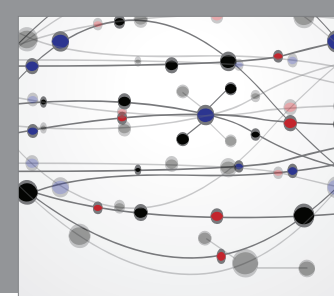

The Scientific World Journal
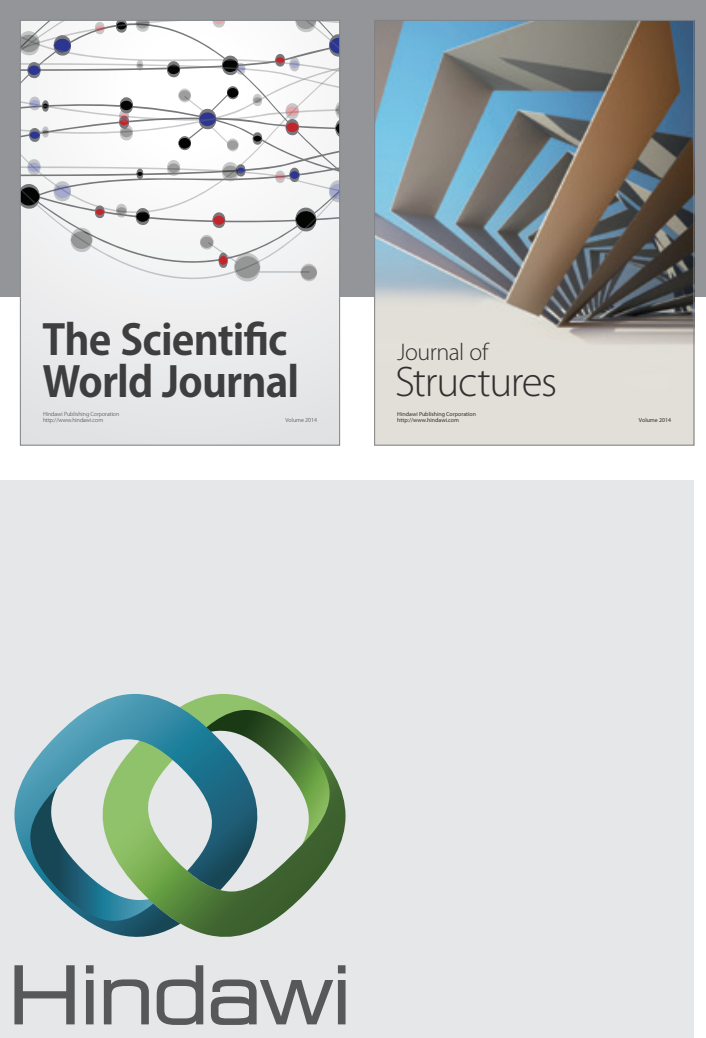

Submit your manuscripts at

https://www.hindawi.com
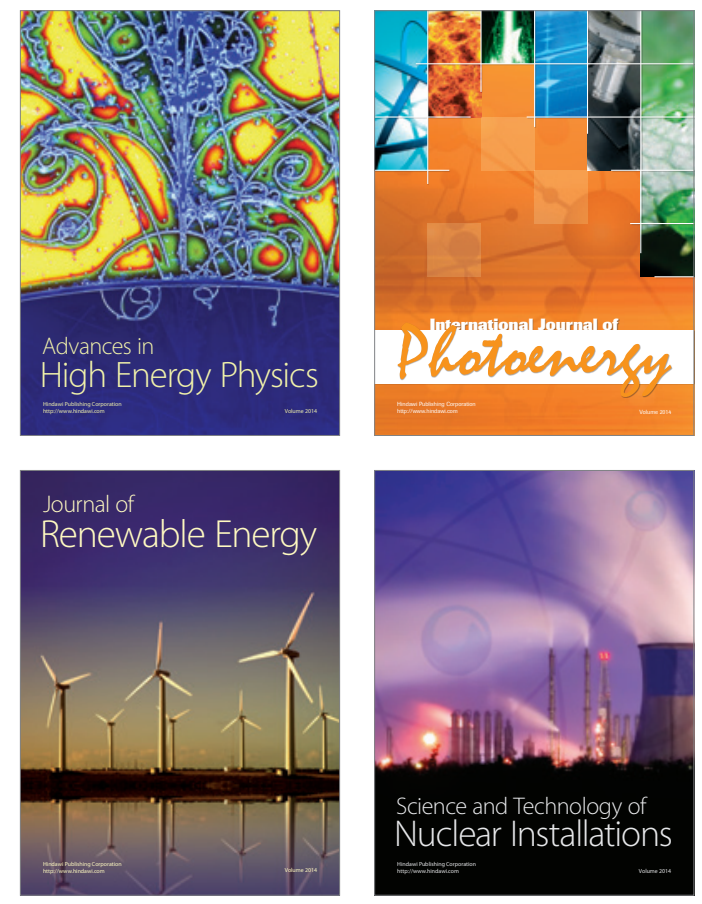
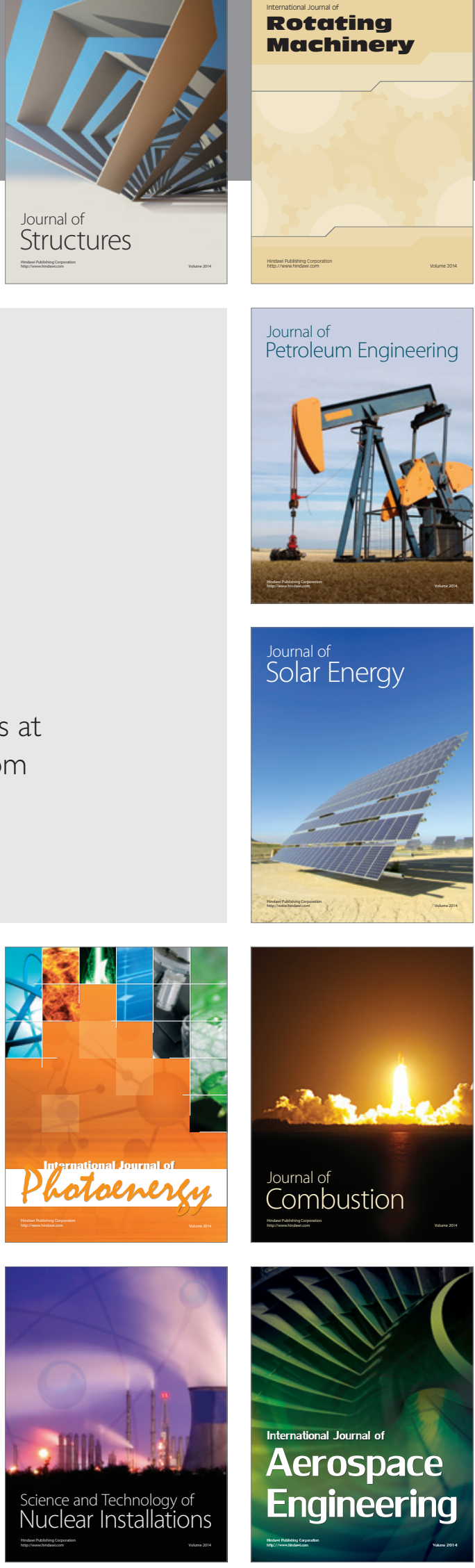